\title{
Not fatal venous air embolism after holmium laser enucleation of the prostate: Case report and review of literature
}

\author{
Daniele Romagnoli ${ }^{1}$, Mobin Ghaemian ${ }^{2}$, Daniele D'Agostino ${ }^{1}$, Paolo Corsi ${ }^{1}$, Marco Giampaoli ${ }^{1}$, \\ Alessandro Del Rosso ${ }^{1}$, Matteo Cevenini ${ }^{3}$, Riccardo Schiavina ${ }^{3}$, Eugenio Brunocilla ${ }^{3}$, Giorgio Davià ${ }^{2}$, \\ Walter Artibani ${ }^{1}$, Angelo Porreca ${ }^{1}$ \\ ${ }^{1}$ Robotic Urology and Mini Invasive Urologic Surgery Unit, Abano Terme Hospital, Abano Terme (PD), Italy; \\ ${ }^{2}$ Anesthesiology and Postoperative Intensive Care Unit, Abano Terme Hospital, Abano Terme (PD), Italy; \\ ${ }^{3}$ Department of Specialistic, Experimental and Diagnostic Medicine, Urology, Alma Mater Studiorum-University of Bologna, \\ S. Orsola Hospital, Bologna, Italy.
}

\begin{abstract}
Summary $\quad$ Objective: Holmium laser has demonstrated high efficacy in urethral disobstruction.

Venous air embolism (VAE) is a rare complication of prostate surgery. Only two cases of venous air embolism (VAE) in patients submitted to HoLEP, have been described. In this paper we show a third case of not fatal VAE after HoLEP. Materials and methods: A case of VAE occurred in holmium laser enucleation (HoLEP) due to obstructive lower urinary tract symptoms (LUTS) in a 70 years old patient. After the procedure, patient's end tidal carbon dioxide $\left(\mathrm{ETCO}_{2}\right)$ levels dramatically decreased at $17 \mathrm{mmHg}$, with pressure airway (PAW)16 mmHg; oxygen saturation level was at 75\%, without any loss in the ventilation circuit and with arterial blood pressure of $94 / 54 \mathrm{mmHg}$. Due to the negativity for other suspicions, the suspect of VAE was postulated.

Result: The immediate switching from laryngeal mask to Oro Tracheal Intubation increased the oxygen level. A cardiac transthoracic ultrasound was negative for air bubbles inside cardiac cavities, without any alteration in the cardiac kinetics. Arterial blood sample turned negative for any alteration compatible with VAE and catheter continuous vesical irrigation was started to obtain clear washing fluid without blood cloths.

The extubated patient showed no neurological defects. Conclusions: An invasive monitoring system is the key to rapidly and correctly identify any embolic episode during this kind of surgery.
\end{abstract}

KEY WORDS: Holmium Laser enucleation of the prostate; Nitrogen embolus; Transurethral resection of the prostate; Urethral disobstruction; Venous embolism.

Submitted 4 August 2019; Accepted 1 September 2019

\section{INTRODUCTION}

Air embolism is a rare but documented complication of prostate surgery. Most of the few cases described are reported to have taken place during transurethral resection of the prostate (TURP). Holmium laser has demonstrated high efficacy in disobstruction, both in cases of bladder neck incision (1) and in a pure enucleative setting. Moreover, Holmium Laser enucleation of the prostate in prostatic adenoma is a technique which provides excellent relief from obstructive symptoms with the advantage of less risk of both bleeding and recurrence.

Since HoLEP can be also applied for obstructive symptoms relief in patients with prostate cancer $(2,3)$, it has gained wide popularity.

So far, only two cases of venous air embolism (VAE) in patients submitted to HoLEP, have been described by Kato (4) and Zhang (5). In the present paper another case of VAE is reported.

\section{Case report}

A 70-year-old patient (172 cm height, $80 \mathrm{Kg}$ weight) was scheduled to HoLEP at our institution due to obstructive lower urinary tract symptoms (LUTS). Serum prostate specific antigen (PSA) was $3.6 \mathrm{ng} / \mathrm{mL}$, digital rectal examination (DRE) was negative for suspicious areas and prostate volume was measured with ultrasound (US) and resulted to be $90 \mathrm{~mL}$. Post void residual volume (PVR) was found to be $200 \mathrm{~mL}$, and preoperatory uroflowmetry showed a $\mathrm{Q}_{\max }$ value of $5.1 \mathrm{~mL} / \mathrm{sec}$.

The patient had been submitted in 2013 to percutaneous coronary angioplasty and coronary stent positioning, and the home therapy consisted of metoprolole, ramipril and cardioaspirin (not preoperatively withdrawn). Preoperative electrocardiogram and chest x-ray were normal, and cardiological evaluation was negative for any modification in the drug scheme. No alterations in preoperative exams were recorded. Standard oxygen monitor was established, and general anesthesia was performed through Superglotty Airway Mask iGel (Intersurgical $^{\odot}$ ) number 4 with gastric tube. Induction was performed with intravenous Propofol (2 mg/Kg and Sufentanyl $0.2 \mu \mathrm{g} / \mathrm{Kg}$ ). Anesthesia was realized through Sevoflurane $(2.0 \%)$ without myorelaxant drugs. Patient was ventilated with mechanical ventilation with tidal volume $7 \mathrm{ml} / \mathrm{kg}$, respiratory rate of $12 \mathrm{rpm}$, and I:E ratio of $1: 1.5$. HoLEP was performed using a $120 \mathrm{~W}$ Ho:YAG VersaPulse ${ }^{\circledR}$ Power Suite ${ }^{\mathrm{TM}}$ laser Source (Lumenis ${ }^{\circledR}$, Santa Clara, California), with a $550 \mu \mathrm{m}$ SlimLine ${ }^{\mathrm{TM}}$ end firing laser fiber. Saline $(0.9 \% \mathrm{w} / \mathrm{v})$ irrigation fluid was used. 
During the procedure patient remained haemodinamically stable without any loss in the ventilation circuit, with a systemic pressure between 110 and $99 \mathrm{mmHg}$, a heart rate of $67 \mathrm{bpm}$, oxygen saturation $99 \%$, end tidal carbon dioxide $\left(\mathrm{ETCO}_{2}\right) 31 \mathrm{mmHg}$ and pressure airway (PAW)16 mmHg. The entire procedure lasted 90 minutes, and before morcellation additional hemostasis with a bipolar loop inserted in a Storz ${ }^{\circledR} 24 \mathrm{~F}$ resectoscope was performed, in order to achieve the highest level of hemostasis in a patient under antiaggregant therapy. No capsular lesion was documented, and morcellation was performed in clear fluid vision, without any bleeding from the surgical field. At the end of the procedure, in order to check for any remnant chip in the bladder, the surgeon filled the bladder with about $80 \mathrm{~mL}$ of air, from the outer sheet of the nephroscope. This procedure usually allows visualization of any remnant prostatic adenoma down piece on the bladder floor, preventing their flooding on the dome, as it usually happens when filling the bladder with saline.

The surgeon found no remnant chips in the surgical field, but immediately after the placement of the urethral catheter, patient's ETCO ${ }_{2}$ levels dramatically decreased at $17 \mathrm{mmHg}$, with PAW 16 and oxygen saturation level decreased at $75 \%$, without any loss in the ventilation circuit and arterial blood pressure of 94/54 mmHg. So laryngeal mask was switched to Oro Tracheal Intubation (OTI), in order to increase the oxygen level. Correct positioning of the tube was confirmed by auscultation via stethoscope. Pulmonary US ruled out the presence of pneumothorax and atelectasia. Due to their negativity, the VAE was suspected: the patient was put in Trendelenburg position and cardiac transthoracic US showed no air bubbles inside cardiac cavities, without any alteration in the cardiac kinesis. Arterial blood sample was collected, but was negative for any alteration compatible with VAE.

After 5 minutes $\mathrm{ETCO}_{2}$ levels spontaneously returned to $40 \mathrm{mmHg}$, with fraction of inspired oxygen $\left(\mathrm{FiO}_{2}\right) 40 \%$ and Oxygen saturation of $100 \%$, and arterial blood sample was negative for any significant alterations $\left(\mathrm{FiO}_{2}\right.$ $100 \%, \mathrm{pH} 7.29, \mathrm{pO}_{2} 217 \mathrm{mmHg}, \mathrm{pCO}_{2} 53 \mathrm{mmHg}$, Sat O2 98\%, acid-base excess ABE-1.9 mmol/L, Lactate level $0.9 \mathrm{mmol} / \mathrm{L}$ ). Catheter continuous irrigation was started, and clear washing fluid without blood cloths was so obtained. The patient was extubated and awakened, and no neurological defects were detected. Another arterial blood sample resulted free from any significant alteration $\left(\mathrm{FiO}_{2} 40 \%, \mathrm{pH} 7.39, \mathrm{pO}_{2} 75.3 \mathrm{mmHg}, \mathrm{pCO}_{2} 40.5\right.$ $\mathrm{mmHg}$, Sat $\mathrm{O}_{2}$ 94.9\%, acid-base excess ABE-0.1 $\mathrm{mmol} / \mathrm{L}$, Lactate level $0.9 \mathrm{mmol} / \mathrm{L}$ ).

Only a conjunctival capillary rupture was detected in the right eye (a sign of augmented endothoracic pressure), but no visus defects were detected. Patient was transferred to the Intensive Care Unit, where a second transthoracic US was performed, together with a serum dosage of myocardial enzymes (Troponine): every analysis was negative for any alteration, so the patient was readmitted to the Urology Ward. Catheter was removed in second postoperative day (POD), while patient was discharged in third POD, without any neurological sequelae.

\section{Discussion}

The advantage of HoLEP over TURP is the excellent hemostatic effect of Holmium laser compared to standard electrical energy. VAE cases have been described in TURP, while, to the present report, only two cases have been described during HoLEP. The first case (4) was due to an incorrect assembly of the morcellator system, with air entry into the endoscopic circuit. The second (5), has left unanswered the source of the gas leading to a fatal massive VAE.

The basis for VAE are the presence of an open venous system and a condition of sub-atmospheric central venous pressure. Moreover (6) air can enter the bloodstream due to active filling: a $5 \mathrm{~cm} \mathrm{H} 2 \mathrm{O}$ pressure gradient is enough to make air enter the opened vessels (7); this condition may occur in HoLEP enucleative phase, when the Surgeon opens perforating vessels, arising from the capsule to the adenoma.

It is important to underline that the entity of VAE is related to the volume of gas entering the venous system. Small size emboli generally have no effect on circulation, thus explaining why there was just a small number of fatal VAE during prostate surgery (7-9). The estimated lethal volume of intravenous air in human circulation is 3-5 mL/Kg (10). There are two possibilities when air enters into the venous bloodstream: if it enters slowly, it can be reabsorbed at the alveolar-capillary interface (11), or, if the air reaches quickly the right heart an acute occlusion of pulmonary artery outflow ensues, leading to fatal VAE. Considering this pathophysiology, we suppose that, in our case, the episode of VAE has been facilitated by air insufflation inside the bladder ("pneumocystoscopy"), to check for any remnant prostatic chip, preventing the flooding of the chips which is caused by suspension in the irrigation fluid. A similar situation had been described when air was inserted through the urinary catheter at the end of the procedure (6) or due to an incorrect assembly of the irrigation system (12) or to an incorrect assembly in the morcellator device (13). In other cases, however, a clear source of air remains either unclear or just postulated: for example, Zhang (5) supposed that fatal VAE had been caused by air entrance into the bladder due to the high-frequency intermittent extraction and insertion of the scope from the sheat during a long lasting procedure (two hours and forty minutes). Chang (14) identified bubbles resulting by water vapor as a possible source of embolism, while Zhang (5) underlined that embolism is linked more likely to air embolus, composed of relatively insoluble nitrogen, than to water embolus resulting from laser absorbance by irrigation fluid.

It is also important to underline that, in this case, the patient did not suffer any damage relatd to VAE and spontaneously fully recovered, while most of the cases of VAE related to prostate surgery are characterized by death of the patient due to massive embolism (7). A possible explanation may be both the amount of air entering the bloodstream, not large, and the absence of any cardiac abnormality as a predisposing factor. An effective strategy to treat VAE consists of immediate placement of the patient in left lateral decubitus (Durant's manouver) maintaining Trendelenburg position, direct aspiration of 
air from the heart via a central catheter and effective cardiopulmonary resuscitation (if required) (5).

As a final consideration, the quality of monitoring is crucial to correctly and directly detect. The correct monitoring scheme is the one adopted in case of general anesthesia, with the possibility of rapidly performing, whenever feasible and possible, transesophageal echocardiography, as reported by Hong (15), who described an incidence of $80 \%$ of VAE during retropubic radical prostatectomy. This procedure remains the actual most sensitive method for detection of air in the hearth, and the recommended monitoring item. In our case, if we had performed the procedure under spinal anesthesia, without invasive monitoring scheme, we might have missed to identify and recognize the embolic episode. Therefore, care must be taken in order to avoid any air entrance in the bloodstream, either directly or indirectly. An invasive monitoring system is the key to rapidly and correctly identify any embolic episode.

\section{REFERENCES}

1. Porreca A, Mineo Bianchi F, D'Agostino D, et al. Ejaculation sparing bladder neck incision with holmium laser in patients with urinary symptoms and small prostates: short-term functional results. Urol Int. 2019; 103:102-107.

2. Noale M, Maggi S, Artibani W, et al. Pros-IT CNR: an Italian prostate cancer monitoring project. Aging Clin Exp Res. 2017; 29:165-172

3. Grasso A, Cozzi G, De Lorenzis E, et al. Multicenter analysis of pathological outcomes of patients eligible for active surveillance according to PRIAS criteria. Minerva Urol Nefrol. 2016; 68:237-41.

4. Kato T, Sugimoto M, Matsuoka Y, et al. Case of vascular air embolism during holmium laser enucleation of the prostate. Int $J$ Urol. 2015; 22:227-9.

5. Zhang W, Ren M. Fatal massive air embolism during holmium laser enucleation of the prostate (HoLEP). Int J Clin Urol. 2017; 6:60-62.

6. Vacanti CA, Lodhia KL. Fatal massive air embolism during transurethral resection of the prostate. Anesthesiology. 1991; 74:186-7.

7. Tsou MY, Teng YH, Chow LH, et al. Fatal gas embolism during transurethral incision of the bladder neck under spinal anesthesia. Anesth Analg. 2003; 97:1833-4.

8. Albin MS, Ritter RR, Reinhart R, et al. Venous air embolism during radical retropubic prostatectomy. Anesth Analg. 1992; 74:151-3.

9. Memtsoudis SG, Malhotra V. Catastrophic venous air embolus during prostatectomy in the Trendelenburg position. Can J Anaesth. 2003; 50:1084-5.

10. Toung TJ, Rossberg MI, Hutchins GM. Volume of air in a lethal venous air embolis. Anesthesiology. 2001; 94:360-1.

11. Presson RG, Kirk KR, Haselby KA, et al. Fate of air emboli in the pulmonary circulation. J Appl Physiol. 1989; 67:1898-1902.

12. Frasco PE, Caswell RE, Novicki D. Venous air embolism during transurethral resection of the prostate. Anesth Analg, 2004; 99:1864-6

13. Kato T, Sugimoto M, Matsuoka $Y$, et al. Case of vascular air embolism during holmium laser enucleation of the prostate. Int $J$ Urol. 2015; 22:227-9.
14. Chang CP, Liou CC, Yang YL, Sun MS. Fatal gas embolism during ureteroscopic holmium:yttrium-aluminiium-garnet laser lithotripsy under spinal anesthesia- a casae report. Minim Invasive Ther Allied Technol. 2008; 17:259-61.

15. Hong JY, Kim JY, Choi YD, et al. Incidence of venous gasembolism during robotic-assisted laparoscopic radical prostatectomy is lower than that during radical retropubic prostatectomy. $\mathrm{Br} \mathrm{J}$ Anaesth. 2010; 105:777-81.

\section{Correspondence}

Romagnoli Daniele, MD (Corresponding Author)

danieleromagnoli87@gmail.com

D'Agostino Daniele, MD

daniele.dagostino@casacura.it

Corsi Paolo, MD

paolo.corsi@casacura.it

Giampaoli Marco, MD

marco.giampaoli@casacura.it

Del Rosso Alessandro, MD

adelrosso@casacura.it

Artibani Walter, MD

prof.artibani@gmail.com

Porreca Angelo, MD

angeloporreca@gmail.com

Robotic Urology and Mini Invasive Urologic Surgery Unit, Abano Terme Hospital Piazza Cristoforo Colombo, 1, 35031 Abano Terme (Padova) (Italy)

\section{Ghaemian Mobin, MD}

mobin.ghaemian@casacura.it

Davia' Giorgio, MD

giorgio.davia@casacura.it

Anesthesiology and Postoperative Intensive Care Unit, Abano Terme Hospital Piazza Cristoforo Colombo, 1, 35031 Abano Terme (Padova) (Italy)

Cevenini Matteo, MD

matteoceve@gmail.com

Schiavina Riccardo, MD

rschiavina@yahoo.it

Brunocilla Eugenio, MD

eugenio.brunocilla@unibo.it

Department of Specialistic, Experimental and Diagnostic Medicine, Urology,

Alma Mater Studiorum-University of Bologna, S. Orsola Hospital

Via Pelagio Palagi, 9, 40138 Bologna, (Italy) 